\title{
Prácticas digitales de estudiantes universitarios hondureños: Estudio de caso
}

\author{
Carlos Joselín, CRUZ GARCÍA \\ María Luisa, ZORRILLA ABASCAL
}

Datos de contacto:

Carlos Joselín Cruz García

Universidad Autónoma del Estado de Morelos

carlos.czgar@gmail.com

María Luisa Zorrilla Abascal

Universidad Autónoma del Estado de Morelos

maria.zorrilla@uaem.mx

Recibido: 03/03/2021

Aceptado: 13/07/2021

\section{RESUMEN}

Este trabajo presenta los resultados de un estudio de caso realizado en la Universidad Pedagógica Nacional Francisco Morazán (UPNFM) en Honduras. Dicho estudio tuvo como objetivo principal identificar y caracterizar las prácticas digitales de los estudiantes fuera y dentro del contexto escolar. Se trabajó desde la postura teórica y metodológica de los Estudios de Nuevos Medios y de los Estudios de Internet, ambas corrientes herederas de los Estudios de Medios (Media Studies). La investigación siguió las líneas metodológicas de la etnografía virtual. Se describen las diferentes técnicas e instrumentos para la recolección de los datos en las dos fases de la investigación: a) la aplicación de un cuestionario y b) la realización de entrevistas a profundidad, así como de e-observación. Se destaca la recuperación de c) un blog (diario digital) como medio para generar reflexiones por parte de los participantes. Los resultados abarcan diferentes aspectos de sus prácticas digitales: redes sociales que más frecuentan; recursos y aplicaciones para actividades de aprendizaje que más usan, hábitos lectores, así como ámbitos en que se manifiestan. Se considera de gran importancia el ahondar en estos temas, ya que no se han realizado investigaciones similares en Honduras y son pocos los estudios de este tipo ubicados en Centroamérica.

PALABRAS CLAVE: prácticas digitales; aprendizaje informal; TIC; etnografía digital 


\title{
Digital practices of Honduran university students: case study
}

\begin{abstract}
This work presents the results of a case study carried out at the Francisco Morazán National Pedagogical University (UPNFM) in Honduras. The main purpose of this study was to identify and characterize the digital practices of students outside and within the school context. We worked from the theoretical and methodological perspective of New Media Studies and Internet Studies, both derivative currents of Media Studies. The research was designed following the methodological principles of virtual ethnography. The different techniques and instruments for data collection in the two phases of the research project are described: a) application of a questionnaire and b) in-depth interviews, as well as e-observation. The retrieval of data was mainly focused through c) a blog (digital diary) as a medium to generate reflections by the participants. The results cover different aspects of their digital practices: most frequently used social media; preferred resources and applications for learning activities, reading habits, as well as areas in which they express themselves. It is considered of great importance to delve into these topics, since no similar research has been carried out in Honduras and there are few studies of this type located in Central America.
\end{abstract}

KEYWORDS: digital practices; informal learning; ICT; digital ethnography 


\section{Introducción}

La ubicuidad de las Tecnologías de la Información y Comunicación (TIC) continúa incidiendo en las prácticas de la sociedad actual, hoy más que nunca. Castells (2000) afirma que tecnología es sociedad y que hoy en día no podemos negar la gran influencia que las Tecnologías de la Información y Comunicación (TIC) tienen en una variedad de manifestaciones y prácticas sociales. En los espacios educativos las TIC se perciben como de gran utilidad para el desarrollo académico de los actores educativos y una de las razones es la fácil adopción que los estudiantes hacen de ellas. Las TIC en la vida de los jóvenes, y de la sociedad en general, han cambiado las prácticas culturales, las formas de interacción y socialización entre pares; la manera en que leen (formato físico o digital), sus creencias, modos de aprendizaje, hábitos y actitudes. A tales manifestaciones, Lévy y Medina (2007) y Casillas y Ramírez (2016) las llaman cultura digital.

Entre algunos de los autores que se han interesado por temas relacionados con prácticas digitales informales y su integración en contextos formales están Parra (2011) en Colombia, Kumar, Liu y Black (2012) en Estados Unidos; Crovi y Lemus (2014), Regil (2014) y Winocur (2015) en México. Los estudios han identificado una diversidad de usos de aplicaciones móviles, de herramientas digitales y de entretenimiento con potencial para el aprendizaje, que van desde el uso de programas de edición de texto hasta programas de software complejos para la edición de videos e imágenes; se han caracterizado también hábitos de lectura entre la pantalla y el libro. Sin embargo, son escasos los estudios que hay al respecto en el contexto centroamericano y en el hondureño específicamente.

Este trabajo es un reporte parcial de los resultados de una investigación más amplia que pretende explicar cómo se manifiesta la cultura digital en el aprendizaje cotidiano de los estudiantes de la Universidad Pedagógica Nacional Francisco Morazán (UPNFM) de Honduras. En el caso específico de este artículo nos interesa explicar cómo son las prácticas digitales en el aprendizaje cotidiano de los antes mencionados. Para tal fin, el texto busca responder a la pregunta: ¿cómo se manifiestan las prácticas digitales en el aprendizaje cotidiano de los estudiantes de la UPNFM? En este momento, en el que las TIC han generado nuevas formas de interacción y de expansión de las dinámicas sociales más allá de los espacios físicos; en donde los actores educativos se encuentran frecuentemente inmersos en el uso de la tecnología en diferentes planos, las barreras entre lo público y lo privado se desvanecen, así como las fronteras entre el espacio educativo y los espacios del hogar, del ocio y del entretenimiento, dando lugar a nuevas oportunidades de aprendizaje.

La cultura digital es una pieza importante dentro de la vida cotidiana de las personas. De acuerdo con NCVER ${ }^{1}$ (2009), la mayoría de la gente está involucrada en algún tipo de aprendizaje informal en donde "las TIC sirven de canal para acceder a la información, pero algunas personas realmente no están conscientes de ello" (p.3)².

\footnotetext{
${ }^{1}$ National Centre for Vocational Education of Australia (Centro Nacional para la Educación Vocacional de Australia).

2 Traducción de los autores.
} 
Cada día estamos mucho más conectados para realizar tareas en general. Según las estadísticas del INE ${ }^{3}$ (2016), respecto a los hábitos de los hondureños en internet, el $55.1 \%$ de la población entre 15 y 29 años usa internet para buscar información, noticias y descarga de software; el 58.1\% para entretenimiento personal; el $58.9 \%$ para comunicarse vía correo electrónico o chat. Mientras que el 32.1\% de la población universitaria usa internet para realizar tareas o estudiar; el $26.8 \%$ para entretenimiento personal y el 65.4\% lo utiliza para comprar productos o servicios.

Al respecto, Willett (2008) sugiere que: "los medios, particularmente los nuevos medios digitales, ofrecen a los jóvenes la oportunidad de ser poderosos y de expresar su creatividad como productores" (p.65). En las redes sociales, no solamente están reafirmando su identidad sino, haciendo amigos de diferentes latitudes con ideas afines; trabajando en equipo; aprendiendo entre pares y por sí mismos; compartiendo proyectos propios: Do It Yourself (Hazlo tú mismo); estableciendo complejas estrategias en equipo para ganar un videojuego y volviéndose expertos en ciertas habilidades dentro de comunidades en línea. En este sentido, el Informe Horizon, realizado por el New Media Consortium (2017), expone que, "aunque tanto los estudiantes como los docentes usan dispositivos móviles con regularidad, siguen necesitando apoyo técnico, logístico y pedagógico de las instituciones para aprender a usar los dispositivos móviles con fines educativos" (p.22). En síntesis, un tema primordial es entender cómo las TIC son utilizadas por jóvenes dentro y fuera de los ambientes educativos y cómo podrían aprovecharse para mejorar el aprendizaje en general, más allá de los centros universitarios.

\section{Cultura digital: Prácticas digitales y aprendizaje informal}

Para llevar a cabo esta investigación se trabajó desde la postura teórica y metodológica de los Estudios de Nuevos Medios (New Media Studies) y de los Estudios de Internet (Internet Studies), ambas corrientes herederas de los Estudios de Medios (Media Studies). Los ENM se enfocan en los dispositivos que posibilitan y extienden nuestras habilidades para comunicarnos, las actividades y prácticas comunicativas que involucran tales dispositivos y los arreglos sociales en torno a dichos dispositivos y prácticas (Lievrouw y Livingston 2006, publicado originalmente en 2002, p. 23).

Para Casillas y Ramírez (2016), la cultura digital es "el conjunto de referentes, técnicas, prácticas, actitudes, modos de pensamiento, representaciones sociales y valores que se desarrollan en torno al ciberespacio y al uso masivo de las computadoras" (p. 37). En este trabajo se entiende cultura digital cuando las personas hacen uso de las TIC y poseen las habilidades y competencias para aprovechar dichas herramientas en todos los aspectos de su vida cotidiana. Por lo tanto, podría decirse que, si una persona no hace uso de las TIC en su día a día, ya sea por ausencia de ellas o que de manera deliberada haya optado por no usarlas, esta persona no tiene o carece de cultura digital. Los autores exponen distintos aspectos o características de la cultura digital de manera muy amplia, aunque en este trabajo nos centraremos en las prácticas digitales de los jóvenes.

\footnotetext{
3 Instituto Nacional de Estadística de Honduras
} 
Para Pires, Masanet y Scolari (2019) las prácticas digitales están relacionadas con el hacer y el discurso que tienen los jóvenes. A grandes rasgos Carriço dos Reis, Rivera, Lopes y Sousa (2018, p.3) comentan que las prácticas digitales son: “acciones y experiencias" mediadas por TIC. Es importante prestar atención a las manifestaciones de las prácticas que tienen que ver con la pertenencia a comunidades virtuales (Jenkins, Purushotma, Weigel, Clinton y Robison, 2009), tales como la multitarea, mayor utilización de internet y telefonía móvil, búsqueda de información. Asimismo, la lectura hipertextual (Winocur, 2015) cada día es más frecuente entre los jóvenes. Las herramientas llamadas informalmente apps como Twitter, Instagram, Snapchat, YouTube, Facebook, Skype, WhatsApp y Google, han ganado popularidad entre la población en los últimos años, especialmente entre los adolescentes, y son las más utilizadas para comunicarse, compartir y descargar contenidos, acceder a la información, mantenerse al tanto de la noticias y tendencias de la moda, etc. Por lo tanto, muchas de las prácticas digitales de los jóvenes están relacionadas con el aprendizaje informal.

El aprendizaje informal se ubica en contextos fuera del entorno educativo, donde la gente aprende sobre temas diversos y a su propio ritmo; al respecto, Scolari señala que "la gente puede participar de varias maneras según sus competencias e intereses porque dependen de enseñanzas entre iguales" (2108, p. 20), estas personas aprenden de manera colaborativa, consensuada y en entornos digitales. En el aprendizaje informal es el aprendiz quien dirige su proceso de adquisición y desarrollo de nuevo conocimiento y, según Asenjo, Asensio y Rodríguez-Moneo (2012), algunos de los elementos que potencian la motivación intrínseca del sujeto en este tipo de aprendizaje son: su libre elección, la disposición a aprender basada en intereses y conocimientos previos, así como la intencionalidad socialmente significativa del aprendizaje, entre otros.

Siendo internet un elemento de la cultura actual, este tipo de aprendizaje podría darse de manera simultánea en los dos ámbitos que poco a poco han borrado sus fronteras entre lo online y offline y ya no representarían una dicotomía. Por su parte, Cobo y Moravec (2011) puntualizan que es cada vez más frecuente que los jóvenes experimenten: "prácticas de aprendizaje formal en entornos informales, y al mismo tiempo que usemos en contextos formales medios antes considerados de carácter informal" (p.25). Esto podría ser el uso de foros de discusión en línea, grupos de Facebook como medio para compartir experiencias de aprendizaje, información, la lectura hipertextual (Winocur, 2015), el FanFic (Lankshear y Knobel, 2008), etc. Por consiguiente, las rutas de aprendizaje de las personas se han ampliado y han generado diversas maneras de interacción con otros en el entorno de la cultura digital. Jenkins et al. (2009) señalan que muchos jóvenes ya están siendo partícipes de:

- [Afiliación] a comunidades en línea (de manera formal e informal) que poseen variadas formas de medios que facilitan la comunicación: Facebook, Twitter, Messenger, Instagram, WhatsApp, juegos en línea, etc.

- Las nuevas formas de producción de contenido en línea como: la creación de videos y la escritura de FanFic, entre otros.

- Colaboración en la resolución de problemas: trabajo en equipos (formales e informales), para realizar tareas y desarrollar conocimiento. Algunos ejemplos que se plantean son Wikipedia, juegos en línea, etc. 
- Circulación: ir con el flujo de los medios como podcast, blog y actualmente los video blogs denominados vlogs. (Jenkins et al., 2009, p.8)

Los jóvenes que experimentan estas prácticas están adquiriendo o desarrollando habilidades respecto al uso de las TIC en varios aspectos de su vida.

\section{Método}

El trabajo aquí reportado se enmarcó en la perspectiva del estudio de caso con técnicas de recolección de datos de la etnografía virtual. Frömming, Köhn, Fox y Terry (2017) explican que los etnógrafos virtuales en la actualidad, en vez de colocar los medios digitales en el centro del análisis, "buscan prestar atención en las variadas y complejas formas en las cuales los ambientes digitales han llegado a ser un aspecto ubicuo de la vida contemporánea y cultural" (p.15).

El muestreo fue intencional, Izcara (2014) expone que el mismo "consiste en la conformación de la muestra mediante la suma de diferentes individuos que se ajustan a una serie de criterios definidos por el investigador" (p.81). Se eligió a los estudiantes de la materia de Taller de Comunicación Educativa, la cual tenía siete grupos el primer trimestre del 2018, ya que la materia es obligatoria para todos los estudiantes de la UPNFM, por lo tanto, había una población heterogénea respecto a su edad, género y carrera. Dicha universidad es la única institución superior pública en Centroamérica que forma docentes para todos los niveles educativos.

Dentro de los criterios teóricos de selección de la muestra se tomaron las premisas de Jenkins et al. (2009); Regil (2014) y el Estudio sobre los hábitos de los usuarios de internet en México realizado por la Asociación de Internet (2017), los cuales establecen que alguien con cultura digital cumple las siguientes características: edad y sexo indistinto; pertenece a comunidades en línea (al menos a una), prefiere leer en formatos digitales antes que en libros tradicionales o gusta de hacerlo en ambos formatos, posee al menos un dispositivo con conexión a internet, busca y analiza las fuentes de información; consume y también produce contenido digital; usa frecuentemente aplicaciones para algún tipo de aprendizaje, usa aplicaciones para comprar (al menos una).

El muestreo se realizó mediante el siguiente proceso: En una fase inicial, se aplicaron 202 cuestionarios, mediante formularios de Google, que tenía dos objetivos: 1) obtener un diagnóstico en términos de las características generales de la población (datos socioculturales, prácticas y habilidades digitales) y 2) seleccionar a ciertos estudiantes que cumplían con los criterio arriba señalados, con la intención de entrevistarlos y conocerlos en profundidad (segunda etapa). Dado que este trabajo es un estudio de caso y los grupos tenían entre 25 y 35 estudiantes fue importante tomar en cuenta que independientemente de cuántos cumplieran el perfil, la capacidad del investigador le permitía solo enfocarse en un total de 6 a 10 estudiantes, que se seleccionarían entre quienes cumplieran el perfil. Se analizaron las respuestas y en la segunda fase, se identificó a un total de 16 estudiantes que cumplía con dichos criterios, pero solamente ocho aceptaron participar en el estudio. El promedio de edad de estos oscilaba entre los 18 y 27, aunque, había un participante de 49 años. Se recabaron consentimientos informados de todos los estudiantes en ambas fases. 
Con los estudiantes seleccionados se llevaron a cabo dos entrevistas en profundidad, una después de aplicar el cuestionario en línea y otra después de la eobservación, con la intención de explorar prácticas, usos y hábitos digitales y despejar dudas. Respecto a la e-observación, llamamos así al seguimiento en línea que se dio a los participantes. Se observaron sus prácticas digitales a partir de información que surgió en la primera entrevista, respecto de las actividades online y offline que realizaban para su aprendizaje en general. Dado que resultó difícil observarlos a todos "en tiempo real", lo que se observó en realidad fueron registros de dos tipos:

a) Los generados en su actividad en línea (como sus publicaciones en redes sociales, por ejemplo). Dicha información fue recolectada en formato de imágenes (capturas de pantalla).

b) Los registros que ellos voluntariamente realizaron en sus blogs o diarios digitales y a los que se dio seguimiento (con recordatorios semanales a través de WhatsApp).

Respecto al tiempo y entradas de los blogs u otros medios, se tomó como referencia el estudio de Beneito-Montagut (2011), quien señala criterios éticos para el seguimiento de un blog. La actividad fue voluntaria, por tal razón, cuatro estudiantes cumplieron con todas las entradas mientras que los otros cuatro solo entregaron parcialmente o nunca las entregaron. Toda la información fue recolectada y transcrita en formato simple del procesador de textos Word y con capturas de pantalla (imágenes) para facilitar su procesamiento y análisis mediante codificación inductiva con ayuda del software Atlas.Ti versión 8.

\section{Resultados}

A partir del procesamiento y análisis de los datos recogidos mediante la eobservación, las entrevistas y el diario digital o blog se describen y caracterizan prácticas y herramientas digitales de los participantes dentro del contexto académico, pero también fuera de éste, con especial interés en las prácticas informales de aprendizaje relacionadas con TIC, ya que, en el proceso de indagación, indirectamente los estudiantes se hicieron más conscientes del uso que les dan a las mismas y cómo podrían explotarlas aún más. Ya que las prácticas digitales de los estudiantes son diversas, se clasificaron por subtemas:

- Aprendizaje informal: el uso de herramientas TIC

- Las redes sociales virtuales

- La lectura: entre el libro y la pantalla

\section{Aprendizaje informal: el uso de herramientas TIC}

YouTube: Una de las actividades informales identificada entre los jóvenes estudiantes fue la reproducción y creación de vídeos en YouTube. Resultados similares han sido encontrados por Padilla, Portilla, y Torres (2020), Rodríguez (2020) y Scolari (2016), quienes señalan a YouTube como una estrategia informal de los estudiantes para aprender dentro del contexto escolar. No obstante, en esta investigación se encontró que el ver/crear vídeos en dicha plataforma estaba más asociado a intereses 
personales de los estudiantes fuera el ambiente educativo y del que muchas veces no eran conscientes (Pires, Masanet, y Scolari, 2019), ya que ellos lo consideraban como un pasatiempo y no como una forma de aprendizaje. Pasatiempos como dibujar, escribir, aprender a tocar un instrumento llevan a los jóvenes a buscar por su cuenta lecciones, canales, videotutoriales y a formarse en las temáticas de su preferencia que, derivado de la e-observación de la muestra, van desde videojuegos, anime, egiptología, cultura india, escritura de poemas y libros, blogs de poesía, dibujo para hacer bocetos de caricaturas, hasta cómo reparar un motocicleta.

En la mayoría de los casos dichas actividades parecen difuminarse entre la rutina cotidiana y los trabajos universitarios o pasatiempos (Cobo y Moravec, 2011), sin que las mismas sean percibidas claramente, tal como lo señala Drotner (2008): "las prácticas de los medios fuera de la escuela, por lo tanto, se centran en procesos y problemas o intereses, y no tanto por estudios sistemáticos de hechos particulares o problemas generales" (p. 169). Algunos jóvenes exponen que dicha herramienta les permite acceder a más contenidos sobre temas que quizás no están disponibles en otros medios; la disponibilidad de ciertos contenidos es algo en los que insisten los estudiantes de esta investigación.

Sin embargo, hubo contrastes respecto al uso de YouTube en actividades de aprendizaje formal, ya que participantes como Mario y Amanda manifestaron nunca haber usado dicho medio en alguna materia con fines educativos $\mathrm{y}$, sin embargo, ambos hacen usos de esa plataforma diariamente para aprender sobre temas que les llaman la atención de manera informal. Aquí sería necesario establecer un "puente" entre las estrategias y los contenidos que los docentes están usando para la enseñanza de ciertos temas que están en la web y que podrían capitalizarse para efectos más formales dentro de las materias.

A diferencia del estudio de Winocur (2015), quien encontró que en los tiempos cotidianos los jóvenes no parecían ser conscientes del uso de dispositivos electrónicos ni aparecían como actividades de ocio en sí, en este estudio con jóvenes de la UPNFM, en las entrevistas sobre sus pasatiempos, en la mayoría de los casos el entrar a YouTube a través de su teléfono fue señalado en reiterada ocasiones como una fuente de entretenimiento, pero también para obtener información o algún tipo de aprendizaje tanto de las materias de la universidad como de temas de intereses personales

Apps: Otra de las actividades digitales más comunes que se encontró fue el uso de algunas aplicaciones (comúnmente llamadas apps) para la creación de contenido de distinta índole más allá de YouTube, tanto como para aprender y afinar sus habilidades en ciertos aspectos o simplemente como pasatiempo. Sin embargo, estas actividades estaban entrelazadas con las materias que cursaban, lo que en algunos casos funcionaba como una manera de aprendizaje informal espontáneo. En este sentido las barreras entre hacer cosas por pasatiempo y aprender se difuminan y podrían potenciarse para objetivos más formales por parte de los docentes universitarios. Derivado de que se identificó que los estudiantes usan diversas herramientas TIC, se describirán solamente las que fueron mencionadas como de uso más cotidiano.

Clip Studio: Jacobo, quien estudia Artes Visuales, en la carrera aprende a hacer diseños, dibujos, ilustraciones, edición de fotografía, etc., con ciertos herramientas 
como Photoshop; sin embargo, también usa otros programas que encontró por su cuenta para aprender a diseñar comics, ya que uno de sus pasatiempos favoritos es leer manga en sitios webs como Tapas, en el que otros fans postean sus creaciones para ser leídos y retroalimentados a su vez por otros, como una especie de foro donde interactúan.

Esta actividad de creación de contenido que Jacobo lleva a cabo en sitios web, llamada FanFic es la que Lankshear y Knobel (2008) atribuyen al millennial y específicamente a los neo millennials y que es llamada prosuming cuando está relacionada con consumo y creación de contenido de un producto cultural específico. Al respecto, cinco de ocho estudiantes del caso practicaban al menos una actividad relacionada con creación de contenido.

Es importante poner de relieve que esta práctica del prosuming requiere conocimiento técnico sobre el manejo de programas de diseño para llevarse a cabo, esto podría llamarse aprendizaje basado en la creación de contenido, y estaría relacionado con lo que Hing y Wang (2015), encontraron como una práctica digital entre los jóvenes universitarios y que llamaron aprendizaje prosuming. No obstante, es importante señalar que, a diferencia de los resultados expuestos por dichos autores, en el caso de los estudiantes de la UPNFM, estos están creando contenido que no está relacionado del todo con un producto cultural en específico, pero sí inspirado en él. Lo anterior con las reservas del caso, ya que no todos los estudiantes de la muestra crean contenido del mismo estilo, otros están más inclinados a la escritura o la música. Sin embargo, podría ser aprovechado en actividades de creación artística en el caso de los estudiantes de la Facultad de Artes de la UPNFM.

Ywritter5: Siguiendo la idea de Lankshear y Knobel (2008), se dice una y otra vez que los jóvenes no leen ni escriben, pero muy a menudo los proyectos de creación de contenido emplean narraciones sofisticadas y complejas, como es el caso de Amanda, quien escribe poemas y prosa de forma aficionada en una red social para fans de mangas japoneses. En su diario digital detalló cómo de manera personal y de forma autogestiva encontró una app que le permite escribir sus narraciones con mayor facilidad. Lo que nos lleva a pensar que los jóvenes leen y escriben de manera diferente en la pantalla. Resultados similares han sido encontrados por Cruz (2016) quien encontró en su estudio maneras de fomentar la lectura entre jóvenes; asimismo, coincide con los estudios realizados en México por Regil (2014) y Winocur (2015) respecto a las prácticas lectoras emergentes entre jóvenes en la Ciudad de México, entre las que destaca una lectura constantemente fragmentaria y no lineal entre la pantalla y los libros impresos.

Padlet: Claudia, estudiante de educación física, explicó que en algunas materias de su carrera usa una app llamada Padlet para realizar sus tareas de manera individual y asincrónica y trabajos de manera colaborativa con sus compañeros de equipo (Jenkins et al., 2009). Dicha aplicación permite invitar a otras personas y trabajar de manera remota en la modificación de un documento o una presentación de PowerPoint (aunque también existe la opción de ser invitado como "solo lector" y "moderador"). A pesar de eso, la app es utilizada también para crear vídeos de índole personal, reafirmando lo expuesto por Cobo y Moravec (2011), quienes puntualizan que es cada vez más frecuente que los jóvenes experimenten: "prácticas de aprendizaje formal en 
entornos informales, y al mismo tiempo que usemos en contextos formales medios antes considerados de carácter informal" (p.25). Esto se refleja en los hábitos cotidianos de los participantes en el estudio.

Amino: Amanda tiene un perfil en la red social llamada Amino, donde se le permite tener un nickname o avatar para personalizar su experiencia; asimismo, la app cuenta con wikis, pequeños cuestionarios o trivias para fans y estadísticas que permiten a los usuarios ver cuántos "me gusta” y cuántos comentarios han recibido sobre un poema, un texto, un vídeo, una imagen, etc., además de que la app alberga una gran cantidad de temas específicos a los que puedes unirte si estás interesado, lo que estaría relacionado con la pertenencia a comunidades virtuales e inteligencia colectiva, términos expuesto por Jenkins et al. (2009) como manifestaciones de la cultura digital. Asimismo, reafirma la idea que los jóvenes buscan aprender entre pares en línea, de manera informal, para volverse expertos en el uso de ciertos software o app y compartir con los demás sus creaciones digitales.

A manera de cierre de este apartado, se puede inferir que los estudiantes de la UPNFM, a través de las entrevistas y del diario digital, señalaron puntalmente las herramientas TIC que utilizan para aprender dentro y fuera del contexto escolar. Cada una de las aplicaciones está estrechamente ligada con sus intereses y pasatiempos, pero también con la carrera que estudian (Cruz, 2020). En el caso específico de los estudiantes participantes de la presente investigación, la intención de usarlas muchas veces es la de potencializar la creatividad artística. Es importante destacar lo anterior porque parece existir una relación entre la creación de contenido orientado a las artes y el uso de herramientas TIC en este grupo de estudiantes en específico; sin embargo, este tema debe ser tratado con mayor profundidad en otros estudios al respecto, ya que no existe suficiente evidencia empírica para generar una premisa al respecto.

\section{Redes sociales virtuales: ¿una red donde no socializo?}

Una de las prácticas digitales que se identifica entre los jóvenes es la pertenencia a comunidades en línea a través de las redes sociales digitales (Hing y Wang, 2015; Kumar, Liu y Black, 2012 y Regil, 2014), donde no solo interactúan entre sí, sino que aprenden y comparten información (Pisani y Piotet, 2009), aunque sus interacciones o su uso varían entre los participantes del estudio. La mayoría de los jóvenes que participaron expresan pertenecer a por lo menos dos redes sociales (las más usadas por los participantes fueron Facebook, Instagram y Twitter), donde comparten gustos o buscan/obtienen información de intereses en común. Derivado de las entrevistas y del diario digital establecimos las siguientes tipologías de uso de las redes sociales.

No uso redes sociales frecuentemente (frecuencia de uso y objetivos personales): en los comentarios tanto de entrevistas, diarios digitales y e-observación, cinco de los estudiantes manifestaron pertenecer al menos a una red social, pero no socializar en ella, esto implica que están allí con un objetivo específico y no entran de manera muy frecuente o tienen limitado el tiempo para permanecer en ella, ya que consideran que les quita el tiempo. En el caso de Miguel y Nicolás, comentaron que no les gusta usar las redes sociales, pero que entienden el valor comunicativo y educativo que podrían tener. 
A pesar de tener una postura crítica frente a las redes sociales digitales y lo que no debería compartirse en ellas, Nicolás también aceptó que ahora se han convertido en una herramienta importante con posibles usos pedagógicos:

“Creo que las redes sociales son importantes, más Facebook que las demás porque son mucho más de ocio, pero Facebook podría aplicarse para propósitos educativos, como vender algo en segunda mano, u otras cosas" (Nicolás, entrevista, abril 2018).

Participo poco o nada en redes sociales digitales por decisión propia (frecuencia de uso y privacidad de la información): Nicolás señala no tener Facebook, ni red social alguna, excepto la app de WhatsApp para comunicarse con sus compañeros de trabajo, grupos de la universidad, familiares y amigos, ya que considera que no las necesita y que se pierde mucha privacidad. Durante la entrevista comentó:

[No uso redes sociales] porque pienso que se pierde un poco el sentido y la magia de la vida privada, de la privacidad, si voy a tomar una foto de lo que estoy comiendo y todos [...] las personas que tampoco son tan cercanas a mí van a verlo, pienso que se pierde un poco la magia de conocer a la gente. (Nicolás, entrevista, febrero 2018)

Una y otra vez se ha señalado la vulnerabilidad de la información y los peligros de los usuarios en redes sociales (Marañón, 2012), en este sentido, los participantes de este estudio parecen ser conscientes de los peligros de compartir demasiadas actividades o tener muchos amigos agregados a sus redes sociales, así que han optado por mantener un "perfil bajo" y con restricciones respecto a lo que comparten, con quién lo comparten y el tiempo que permanecen en las mismas. Esto parece estar relacionado con la edad de los participantes, ya que son jóvenes universitarios, que tienen acceso a información respecto al peligro que las redes sociales digitales podrían conllevar.

Migración a redes sociales (uso de redes sociales y control de la interacción social): siguiendo la idea anterior, respecto a la falta de privacidad en redes sociales digitales, algunos de los estudiantes han optado por migrar a otras menos interactivas o como los mismos estudiantes las llaman "con menos presión social, más privadas". Lorena señaló que ella prefiere Twitter porque es "una red social donde no socializo" (diario digital, febrero 2018). Dicha estudiante prefiere esa plataforma porque puede publicar sin sentirse abrumada por contestar los mensajes o tener que ver constantemente "los mismos memes una y otra vez", inclusive pone de relieve que ella notó cómo los memes sobre asuntos políticos o sobre una noticia tiene un efecto dominó entre las personas.

\section{La lectura: entre el libro y la pantalla}

Una de las prácticas que se identificó entre los estudiantes del caso fue la lectura fragmentada y no lineal (Cruz, 2020), que abarca la tradicional, la que se hace en papel, hojas sueltas, folletos y fotocopias, pero también la que se hace en soportes digitales. Estas prácticas no son excluyentes y los jóvenes van y vienen entre la pantalla y los libros tradicionales, y más bien están determinadas por el objetivo que estén persiguiendo en ese momento.

A pesar de que para muchos jóvenes leer tuits, blogs, páginas web y publicaciones en Facebook, no es considerado como lectura (Winocur, 2015), para los estudiantes participantes en este estudio de caso sí están conscientes y describen sus prácticas 
cotidianas entre la pantalla y el libro tradicional de manera muy específica en sus diarios digitales. Prácticas lectoras que van desde el manga, cómics, archivos PDF para sus materias, copias, apps y sitios web de interés. Esto podría ser debido a que, en muchos casos los jóvenes relacionan el concepto de lectura solamente cuando se refiere al libro de carácter académico u obras literarias consideradas canónicas. A continuación, se detallan algunas prácticas lectoras de los estudiantes de la UPNFM:

Lectura en la pantalla sobre cuestiones técnicas: está enfocada a entender programas de computadora sofisticados para llevar a cabo tareas como diseño de dibujos para cómics y diseños complejos de arquitectura. Jacobo y Miguel alternan entre las lecturas por placer y una lectura mucho más técnica, es decir, de manuales para el uso de software especializado. En este sentido, es más fácil encontrar los textos en formato PDF que en físico, ya que son manuales para realizar tareas muy específicas. Los libros impresos los prefieren para novelas, especialmente de ciencia ficción, cómics y manga.

La lectura en pantalla por disponibilidad de contenido: en el caso de Jacobo, escribió en su diario digital que le gusta mucho leer, pero que prefiere leer en línea o en formato PDF debido a que ciertas temáticas no están disponibles en impreso, tales como algunos cómics o mangas que son difíciles de conseguir, son caros o tardan mucho tiempo en llegar al país. Asimismo, señaló que muy pocas veces lee en formato físico y que no le gusta ir a la biblioteca. Aunque su interés lector se centra en los textos de diseño visual, lo que más lee está relacionado con guías para el manejo de software específico para diseño de dibujos y para mejorar sus viñetas para webcómics.

Lectura en la pantalla por motivos académicos: este tipo de lectura usualmente se hace en formato PDF, cuando el docente comparte en algún grupo de WhatsApp la lectura para las clases. Sin embargo, esto depende muchas veces de cómo los docentes proporcionan los textos a los estudiantes, en la mayoría de los casos es en las tradicionales fotocopias. Lorena, estudiante de enseñanza del inglés, indicó una distinción en sus preferencias lectoras respecto a las que hace por placer y las que hace en algunas materias. Usualmente cuando tiene que leer para alguna materia busca el texto en PDF y lo lee en pantalla, pero cuando se trata de una lectura por placer, el formato en papel siempre es su primera opción, también lee textos académicos en formato impreso.

Es importante puntualizar que la participante no se cierra a la lectura digital, pero sus rutinas de aprendizaje están plenamente ligadas a la lectura en papel con la cual se siente mucho más cómoda a la hora de estudiar. Asimismo, comentó que los lugares favoritos para leer son tanto la biblioteca de la UPNFM, como los lugares al aire libre (parques, la terraza de su casa).

Lectura en la web por placer: esta lectura tiene que ver con cómics, manga, poemas, o que se lee en comunidades virtuales específicas como Amino, o sitios para fanáticos de los cómics como Tapas, que no está disponible en formato físico y que obedece a una lectura con el fin de disfrutarla. Amanda y Jacobo se encuentran en este grupo de lectores en pantalla. Aquí es importante poner de relieve que frecuentemente los jóvenes en las entrevistas comentaron que usan la lectura en pantalla por razones económicas, ya que comprar libros es costoso, y muchas veces ni siquiera están disponibles en las tiendas, por lo cual algunos han optado por comprarlos en formato 
e-pub vía Instagram, como lo hace Lorena. Es preciso señalar que las lecturas que aquí describimos pasan a veces de manera simultánea, en ocasiones hipertextual, de acuerdo con los gustos de los lectores. Esto está relacionado con la lectura hipertextual que es mencionada por Regil (2014), como uno de los signos de la cultura digital de los jóvenes universitarios.

A manera de cierre, se infiere que las lecturas de los jóvenes dentro y fuera del contexto educativo están entrelazadas y que cambian de acuerdo con el objetivo que se persiga. Asimismo, los estudiantes del caso de la UPNFM tienen bien identificadas los sitios y aplicaciones donde prefieren leer, estos sí relacionan el manga, cómics, y publicaciones en Twitter como lecturas, lo que podría tener potencial educativo si se establecen estrategias para potenciar el gusto lector en pantalla por parte de algunos estudiantes y profesores.

\section{Discusión y conclusiones}

El objetivo de este artículo es presentar una visión panorámica de las prácticas digitales de los estudiantes hondureños, en formación docente, en el aprendizaje fuera y dentro de la universidad. Se encontró que dichas prácticas son variadas debido a los propios hábitos diarios, rutinas de trabajo e intereses específicos. No obstante, los jóvenes tuvieron puntos de encuentro respecto a ciertas herramientas que usan constantemente para estudiar, trabajar, aprender por su cuenta o en equipo, por ejemplo, YouTube que destaca como uno de los principales sitios web que usan para buscar información (un 17\% de la muestra total señaló usar dicha plataforma para ver tutoriales). Esta es una de las herramientas que coincide con los resultados de otras investigaciones (Pires, Masanet y Scolari, 2019) como una de las más usadas por los jóvenes para aprender de manera informal. No obstante, no hay todavía estrategias didácticas por parte de los docentes para sacar el mayor provecho a las habilidades de creación de contenido que algunos estudiantes demuestran dominar en dichas plataformas.

Los estudiantes comentaron la participación en foros de discusión para aprender a dibujar/editar caricaturas de manera más profesional y tomar cursos en línea como en Coursera, por ejemplo. Es importante destacar que los ocho estudiantes del caso mencionaron el uso de Google traductor (15\%) y Wikipedia (15\%) y solo $11 \%$ usa programas de ofimática. Estos resultados difieren de los encontrados por Kumar, Liu y Black (2012) en Estados Unidos, donde las aplicaciones más usadas fueron Blogs (60\%), Google Docs (42.2\%) y Wikis (47.7\%). El uso frecuente de Google traductor en los estudiantes obedece a que generalmente hacen búsquedas de información, cómics, libros, artículos que no están disponibles en español y que tiene que traducir para poder hacer uso del material, cuestión que no ocurre con los estudiantes en Estados Unidos de América, ya que su lengua nativa es el inglés y, por lo tanto, no tienen que usar dicha herramienta de manera tan frecuente. También se encontró entre los estudiantes de esta investigación el uso del blog y Padlet como herramientas que los jóvenes usan en contextos formales e informales para realizar análisis respecto a contenidos educativos, escribir poemas, textos reflexivos sobre su vida, escribir libros, hacer reseñas sobre libros y películas. 
Lo anterior podría relacionarse con lo expuesto por Cobo y Moravec (2011), quienes sostienen que muchas de las competencias y habilidades que las personas utilizan a diario no son aprendidas en los centros de enseñanza. Esto es que en muchas ocasiones las personas adquieren habilidades digitales haciendo "otras cosas" en entornos de socialización y de manera no inducida. Por lo tanto, se sugiere que estudiantes y docentes podrían hacer curaduría de contenido en YouTube para así generar una especie de catálogo de vídeos que podrían usarse en algunas materias o incluso incrustados en Moodle, o generar vídeos ad hoc para una determinada materia o contenido.

Respecto al uso de internet, 33\% de los estudiantes señaló usar internet con fines educativos, lo que contrasta con lo encontrado por Regil (2014) en México, donde solamente el 2\% usa internet con fines de creación de material didáctico. Un $24 \%$ de los estudiantes de la UPNFM en Honduras usa internet para fines de entretenimiento, un porcentaje mucho menor comparado con lo que Parra (2011) encontró entre los estudiantes colombianos, ya que reportó que un $78 \%$ de los estudiantes usaban internet con fines de entretenimiento.

Otra práctica común entre los estudiantes fue la lectura, aunque se encontró que los jóvenes usualmente leen en formato impreso, en físico, más que en la pantalla, un $38 \%$ de la población comentó leer en formato impreso. Pero también se hizo visible que navegan entre la pantalla y el formato impreso de acuerdo con el objetivo que persiguen con la lectura. Un $56 \%$ de los jóvenes lee en pantalla en diferentes dispositivos como tablets, teléfonos móviles, computadoras portátiles y de escritorio. Esta información contrasta con lo que encontró Regil (2014) en México, donde el 11\% lee en línea todo el tiempo; esto es relevante porque la lectura en formato impreso parece ser todavía preferida por los jóvenes. A diferencia de los lectores de la Ciudad de México que señala Winocur (2015), los estudiantes de la UPNFM sí son conscientes de que sus prácticas de lectoescritura en pantalla y en físico son actividades que cruzan los ámbitos académicos y escenarios informales; asimismo, los estudiantes están estrechamente involucrados en actividades de lectoescritura como blogs, libros, poemas, guiones de cómics, etc. Cabe destacar que también se encontró que la inseguridad en el país impide que puedan leer en pantalla en áreas públicas, lo que modifica sus prácticas lectoras, ya que se ven obligados a imprimir en papel los textos.

Una tercera práctica digital que se identificó en los participantes fue la pertenencia a redes sociales, siendo Facebook (20\%), YouTube (18\%) e Instagram (11\%) las más usadas. Similares resultados fueron encontrados por Kumar, Liu y Black (2012), Hing y Wang (2015) y Regil (2014). No obstante, se pudo apreciar tanto en las entrevistas como en el diario digital, que Facebook está decayendo en popularidad entre los jóvenes y que están migrando a Instagram, ya que según los estudiantes se sienten más seguros y cómodos con la privacidad de dicha aplicación brinda, a diferencia de Facebook, donde sí tiene una cuenta, pero no están regularmente navegando.

Por otro lado, un aspecto particular que destaca en los resultados encontrados en esta investigación fue que el uso y acceso a dispositivos digitales en Honduras está condicionado y coartado por la inseguridad, que no permite que los jóvenes se movilicen con sus dispositivos de manera libre y segura, lo que deriva en que las prácticas digitales de los hondureños se vean modificadas y adaptadas a un entorno 
donde permea el peligro. Según Insight Crime (2019) Honduras está en el quinto puesto de países más inseguros de Latinoamérica. Lo anterior refleja que el uso de las tecnologías en países con altos índices de inseguridad es diferente al que se registra en los países que no presentan tanta incidencia delictiva.

Los estudiantes del caso expusieron a lo largo de las entrevistas, las diferentes maneras en que este problema les afecta desde la imposibilidad de poder llevar sus computadoras portátiles a la universidad, el no poder leer en sus tablets o celulares en el transporte público por miedo a ser asaltados y optar por leer en copias; no poder tomar materias donde se les pide que traigan consigo un equipo, y no poder conectarse en sitios de acceso público por la falta de garantías a su seguridad personal. Por consiguiente, todo lo que se ha teorizado en países desarrollados respecto a las TIC tiene que ver con contextos completamente diferentes a los que se viven en Latinoamérica. La inseguridad inhibe el uso de la tecnología de manera fluida y espontánea, los estudiantes de la UPNFM, y la población en general, se privan de ciertos beneficios de las TIC en su día a día por no arriesgarse a perder sus dispositivos.

Cabe señalar que la UPNFM cuenta con el Centro de Acceso a la Información (CAI), donde los estudiantes pueden usar computadoras, tablets para desarrollar sus actividades académicas, lo que ha sido un gran aporte para paliar el problema antes señalado, aunque dicho Centro no es tan amplio para atender a la población total de la UPNFM. Finalmente, hay que puntualizar que esta brecha de (in)seguridad no se contempla en los estudios sobre desigualdad digital, esto porque es inexistente o menos visible en los países desarrollados. Se considera importante este elemento, ya que la presencia de brechas digitales entre los jóvenes modifica sus prácticas respecto a las TIC, como en el caso de los estudiantes de la UPNFM.

En general, las prácticas digitales de los estudiantes de la UPNFM están ampliamente difuminadas entre los contextos informales y formales, lo que trae consigo que su uso sea altamente frecuente y fluido; no obstante, se considera que es importante que los jóvenes sean dirigidos por los docentes respecto a la exploración y explotación de las herramientas TIC, ya que en muchas ocasiones las experiencias de aprendizaje podrían no tener contornos claros para los jóvenes.

\section{Limitaciones y prospectiva del estudio}

Si bien uno de los aportes principales del estudio es su contexto, en Honduras, país del que se ha escrito muy poco en el tema objeto del presente artículo, cabe reconocer sus limitaciones en cuanto a la cobertura de la investigación, pues al ser un trabajo doctoral, se trató de un estudio realizado por una persona con presupuesto y tiempo restringidos. Asimismo, la disponibilidad de las personas participantes limitó en cierta medida el volumen de datos que fue posible recoger de sus prácticas cotidianas, a partir de la metodología del diario digital.

Las principales áreas de oportunidad que se vislumbran para dar continuidad a los hallazgos de este estudio son tres: 1) La inseguridad ciudadana como factor que incide en las prácticas de uso de TIC, la cual hemos tipificado como una más de las brechas digitales: la brecha de (in)seguridad; 2) La tipificación y caracterización de herramientas TIC que usan los estudiantes en su aprendizaje informal para que puedan 
capitalizarse de forma efectiva en contextos formales; 3) Mayor exploración de metodologías de investigación híbridas, que combinan técnicas tradicionales de acopio de datos y e-metodologías, en especial aquellas que implican un involucramiento activo del participante, como el diario digital.

\section{Agradecimientos}

Este trabajo fue parcialmente financiado por la beca CLACSO-CONACYT para extranjeros en México y la beca CONACYT del Programa Nacional de Posgrados de Calidad.

\section{Conflicto de intereses}

Los autores declaran no tener ningún conflicto de intereses.

\section{Referencias}

Asenjo, E., Asensio, M., y Rodríguez-Moneo, M. (2012). Aprendizaje informal. En Asensio, Rodríguez, Asenjo y Castro (Eds.). Museos y Educación. Series Iberoamericanas de Museología, año 3, volumen 2 (pp. 39-53). Recuperado de: https://repositorio.uam.es/bitstream/handle/10486/11469/57000_3.pdf?seq uence $=1$

Asociación de Internet de México. (2017). 13 estudio sobre los hábitos de los usuarios de Internet en México, 2017, Infotec.

Beneito-Montagut, R. (2011). Ethnography goes online: towards a user-centred methodology to research interpersonal communication on the internet. Qualitative Research,11(6), 716-735. Doi: https://doi.org/10.1177/1468794111413368

Carriço dos Reis, B. M., Rivera Magos, S., Lopes, P., y Sousa, J. (2018). Prácticas digitales de los jóvenes portugueses y mexicanos. Un estudio comparativo. index. Comunicación, 8(3), 207-227.

Casillas, M. A. (2016). Conferencia Magistral: La incorporación de las TIC a la Educación Superior. Presentada en el XI Encuentro Iberoamericano de Educación, Veracruz, Veracruz.

Casillas Alvarado, M. A. y Ramírez Martinell, A. (2016). El Habitus Digital: Una Propuesta para su Observación. En Coloquio Haciendo trabajar a Pierre Bourdieu desde América Latina y el Caribe: Habitus y campo en la investigación social, realizado por el Instituto de Investigaciones Sociales de la UNAM. [Archivo PDF] https://www.uv.mx/personal/mcasillas/files/2016/10/habitusdigital-

Coliquio-Bourdieu.pdf

Castells, M. (2000). Globalización, sociedad y política en la era de la información. Revista Análisis Político, No 37, abril-junio de 1999, 2-17. https://dialnet.unirioja.es/servlet/articulo?codigo=4008342

Cobo-Romaní, J. C. y Moravec, J. (2011). Aprendizaje invisible: Hacia una nueva ecología de la educación. Edicions Universitat.

Cruz, C. (2016). La experiencia de lectura de una novela transmedia en estudiantes de una escuela secundaria en Cuernavaca [tesis de maestría, Universidad Autónoma del Estado de Morelos]

Cruz, C. (2020). La cultura digital en el aprendizaje de estudiantes de la Universidad 
Pedagógica Nacional Francisco Morazán[tesis doctoral, Universidad Autónoma del Estado de Morelos]

Drotner, K. (2008). Leisure is hard work: Digital practices and future competencies (pp. 167-184). MacArthur Foundation Digital Media and Learning Initiative.

Frömming, U. U., Köhn, S. Fox, S., y Terry, M. (2017). Digital Environments: Ethnographic Perspectives Across Global Online and Offline Spaces. Medienwissenschaft.

Insight Crime. (22 de Enero de 2019). Balance de InSight Crime sobre los homicidios en 2018. Insight Crime. Recuperado el 20 de julio del 2020 de https://es.insightcrime.org/noticias/analisis/balance-de-insight-crime-sobreloshomicidios-en-2018/

Instituto Nacional de Estadística, I.N.E. (2016). Acceso a tecnologías de información y comunicaciones

(TIC).

http://170.238.108.227/binhnd/RpWebEngine.exe/Portal?BASE=EPH2016\&la $\mathrm{ng}=\mathrm{ESP}$

Izcara, S. (2014) Manual de investigación cualitativa. Fontamara.

Jenkins, H., Purushotma, R., Weigel, M., Clinton, K., y Robison, A. J. (2009). Confronting the challenges of participatory culture: Media education in the 21st century. MA: The MIT Press.

Kumar, S. y Liu, F. y. (2012). Undergraduates' collaboration and integration of new technologies in higher education: Blurring the lines between informal and educational context. Digital culture and Education, pp.248-259.

Lankshear, C. y Knobel, M. (2008). Nuevos alfabetismos. Su práctica cotidiana y el aprendizaje en el aula. Segunda ed. Morata.

Lévy, P. y Medina, M. (2007). Cibercultura: informe al Consejo de Europa. Ciencia, Tecnología y Sociedad, 16, 230.

Lievrouw, L. y Livingston, S. (2006). Introduction to the Updated Student Edition. En: L. Lievrouw y. S. Livingstone, (Eds). The Handbook of New Media. Updated Student Edition. Segunda ed. Sage Publications, pp. 1-14.

Marañón, C. O. (2012). Redes sociales y jóvenes: Una intimidad cuestionada en internet. Aposta. Revista de Ciencias Sociales, (54), 1-16.

NCVER. (2009). Informal learning at glance. [Archivo PDF] https://ala.asn.au/public/docs/report/Informal_learning-At_a_glance.pdf

New Media Consortium. (2016). Horizon Report > 2016 Higher Education Edition. http://cdn.nmc.org/media/2016-nmc-horizon-report-he-EN.pdf

Parra, E. (2011). La cultura digital de los estudiantes universitarios en entornos académicos. Signo y pensamiento, 144-155.

Padilla, E. J., Portilla, G. I., y Torres, M. (2020). Aprendizaje autónomo y plataformas digitales: el uso de tutoriales de YouTube de jóvenes en Ecuador. Estudios pedagógicos (Valdivia), 46(2), 285-297.

Pires, F., Masanet, M. J., y Scolari, C. A. (2019). What are teens doing with YouTube? Practices, uses and metaphors of the most popular audio-visual platform. Information, Communication \& Society, 117.

DOI:10.1080/1369118X.2019.1672766

Pisani, F. y Piotet, D. (2009). La alquimia de las multitudes: cómo la web está cambiando el mundo. Paidós. 
Regil, L. (2014). Cultura digital universitaria. [Tesis doctoral, Universitat Autònoma de Barcelona].

Rodríguez, E. R. (2020). Tutoriales de Youtube como estrategia de aprendizaje no formal en estudiantes universitarios. RIDE Revista Iberoamericana para la Investigación y el Desarrollo Educativo, 11(21).

Scolari, C. A. (2016). Alfabetismo transmedia: Estrategias de aprendizaje informal y competencias mediáticas en la nueva ecología de la comunicación= Transmedia literacy: informal learning strategies and media skills in the new ecology of communication. Telos: Revista de pensamiento sobre Comunicación, Tecnología y Sociedad, 193, 13-23.

Scolari, C. A. (2018). Introducción: del alfabetismo mediático al alfabetismo transmedia. En: Scolari, C. A. (Ed.) Adolescentes, medios de comunicación y culturas colaborativas. Aprovechando las competencias transmedia de los jóvenes en el aula. Recuperado de: http://transmedialiteracy.upf.edu/sites/default/files/files/TL_Teens_es.pdf

Thompson, J. (1993). Ideología y Cultura Moderna. Teoría crítica social en la era de la comunicación de masas. UAM Xochimilco.

Willett, R. (2008). Consumer Citizens Online: Structure, Agency, and Gender in Online Participation en David Buckingham (Ed.), Youth, Identity, and Digital Media. The John D. and Catherine T. MacArthur Foundation Series on Digital Media and Learning. The MIT Press, 2008. 49-70. doi:10.1162/dmal.9780262524834.049

Winocur, R. (2015). Prácticas tradicionales y emergentes de lectoescritura en jóvenes universitarios. En García Canclini, N., Gerber, V., López, A. Nivón, E., Pérez, C., Pinochet, C. y Winocur, R. (eds). Hacia una antropología de los lectores (pp. 243281). Ediciones Culturales Paidós, Fundación Telefónica y Universidad Autónoma Metropolitana. 